Article

\title{
Endothelium-Independent Vasorelaxant Effect of Ligusticum jeholense Root and Rhizoma on Rat Thoracic Aorta
}

\author{
Bumjung Kim, Kyungjin Lee, Khanita Suman Chinannai, Inhye Ham, Youngmin Bu, \\ Hocheol Kim and Ho-Young Choi * \\ Department of Herbology, College of Korean Medicine, Kyung Hee University, Seoul 130-701, Korea; \\ E-Mails: ori-pharm@hanmail.net (B.K.); dostudy@naver.com (K.L.); \\ sumankhanita6@gmail.com (K.S.C.); iham@khu.ac.kr (I.H.); ymbu@khu.ac.kr (Y.B.); \\ hckim@khu.ac.kr (H.K.) \\ * Author to whom correspondence should be addressed; E-Mail: hychoi@khu.ac.kr; \\ Tel.: +82-2-961-9372; Fax: +82-2-965-9372.
}

Academic Editor: Derek J. McPhee

Received: 23 April 2015 / Accepted: 8 June 2015 / Published: 10 June 2015

\begin{abstract}
Ligusticum jeholense has been used as the traditional medicine 'Go-Bon' (Chinese name, Gao-ben) in China and Korea. Considering the increased use of medicinal herbs to treat hypertension, in this study, we aimed to investigate the mechanisms of the vasorelaxation effect caused by L. jeholense. We tested the methanol (MeOH) extract of L. jeholense root and rhizoma for vasorelaxant effects; while using an isolated organ-chamber technique, L. jeholense extract (LJE) induced relaxation in the rat aortic rings by stimulating vascular endothelial and smooth muscle cells. LJE showed concentration-dependent relaxant effects on endothelium-intact and endothelium-denuded aortic rings pre-contracted with both phenylephrine $(\mathrm{PE})$ and potassium chloride $(\mathrm{KCl})$ in $\mathrm{Krebs}-\mathrm{Henseleit}(\mathrm{KH})$ buffer. The vasorelaxant effect of LJE was partly attenuated by pre-treatment with glibenclamide or 4-aminopyridine (4-AP) as $\mathrm{K}^{+}$channel blockers. Moreover, LJE showed concentrationdependent inhibition of vasoconstriction by $\mathrm{Ca}^{2+}$ supplementation in the aortic rings that were pre-contracted with $\mathrm{PE}$ or $\mathrm{KCl}$ in $\mathrm{Ca}^{2+}$-free $\mathrm{KH}$ buffer. In addition, a combination of LJE and nifedipine, pre-incubated further, decreased PE-induced contractions. The results suggested that LJE-induced vasorelaxation were related to blocking $\mathrm{K}^{+}$channels and inhibiting entry of extracellular $\mathrm{Ca}^{2+}$ via receptor-operative $\mathrm{Ca}^{2+}$ channels (ROCCs) or voltage-dependent $\mathrm{Ca}^{2+}$ channels (VDCCs).
\end{abstract}


Keywords: Ligusticum jeholense; vasorelaxant effect; potassium channels; calcium channels; hypertension

\section{Introduction}

High blood pressure, or hypertension, is a major determinant of mortality caused by cardiovascular disease, cerebrovascular disease, and stroke. In hypertension, vasoreactivity is an inevitable factor for the treatment of hypertension because it affects circulation and blood pressure in the cardiovascular system. Quality of life is important for antihypertensive therapy. However, although these drugs continue to be developed to treat hypertension, synthetic drugs have various adverse effects. The efficacy of these drugs increases in a dose-dependent manner, which leads to more adverse effects [1]. In addition to synthetic drugs, the use of herbs or herbal extracts is increasing in China, Japan, and Korea [2]. Recently, the use of natural herbs has shown a steady growth because of low toxicity and well-established therapy [3]. Many plants used in traditional medicine have been investigated for treating cardiovascular disease [4].

The genus Ligusticum (Umbelliferae), consisting of approximately 50 species growing in Asia, Europe, and North America, is complex and widespread [5]. The root and rhizoma of Ligusticum species are generally used in traditional medicine in China, Japan, and Korea to treat headaches, arthralgia [6], pain, common cold [7], and cardiovascular disease such as angina pectoris [8]. Recent studies have shown that these medicinal plants have anti-infective, sedative, analgesic [9], anti-cancer [10], anti-inflammatory [11], anti-mycobacterial [12], anti-nociceptive activity [13], anti-neuroinflammatory [14], and vasorelaxation effect [8].

Ligusticum jeholense Nakai et Kitagawa has been used as the traditional medicine, 'Go-Bon,' (Chinese name, Gao-ben) in China and Korea. Seven compounds in the root and rhizoma of L. jeholense were identified, such as levistolide A., xiongterpene, linoleic acid, sucrose, daucosterol, ferulic acid, and beta-sitosterol [15]. The main components from the L. jeholense essential oil were isolated, such as $m$-diaminobenzene, ligustilide [7], and $\beta$-phellandrene [5].

There are only a few pharmacological studies about this medicine, describing its anti-bacterial and anti-oxidant activity [7]. Although vascular activity was previously reported for the essential oil of L. jeholense [16], no study has investigated the vasorelaxation effects caused by L. jeholense.

Thus, considering the increased use of medicinal herbs to treat hypertension, in this study, we aimed to investigate the mechanisms of the vasorelaxation effect caused by L. jeholense. We found that the methanol $(\mathrm{MeOH})$ extract of L. jeholense root and rhizoma (LJE) has vasorelaxant effects; while using an isolated organ-chamber technique, LJE induced relaxation in the rat aortic rings by stimulating vascular endothelial and smooth muscle cells. To our knowledge, this is the first report to demonstrate the vasorelaxation mechanisms of $L$. jeholense in Korea. 


\section{Results and Discussion}

\subsection{Effect of LJE on Phenylephrine (PE)- or Potassium Chloride (KCl)-Induced Contraction}

We investigated the concentration-dependent vasorelaxant effects of LJE $(30-500 \mu \mathrm{g} / \mathrm{mL})$ on endothelium-intact aortic rings pre-contracted with $\mathrm{PE}(1 \mu \mathrm{M})$ or $\mathrm{KCl}(60 \mathrm{mM})$ in standard Krebs-Henseleit (KH) buffer. The vasorelaxant effect of LJE was calculated as a percentage of the relaxation in response to $\mathrm{PE}$ and $\mathrm{KCl}$ on the aortic rings.

LJE caused concentration-dependent relaxation in endothelium-intact aortic rings pre-contracted by $\mathrm{PE}$ or $\mathrm{KCl}$ treatment. The maximal relaxant effect was $76.4 \pm 1.2 \%$ and $94.6 \pm 4.5 \%$ at the concentration of LJE $(500 \mu \mathrm{g} / \mathrm{mL})$, respectively (Figure 1). In this study, we found the optimal concentration to generate a complete dose-response for LJE by studying the results of several tests. We applied the same concentration and equilibration time throughout the experimental period.
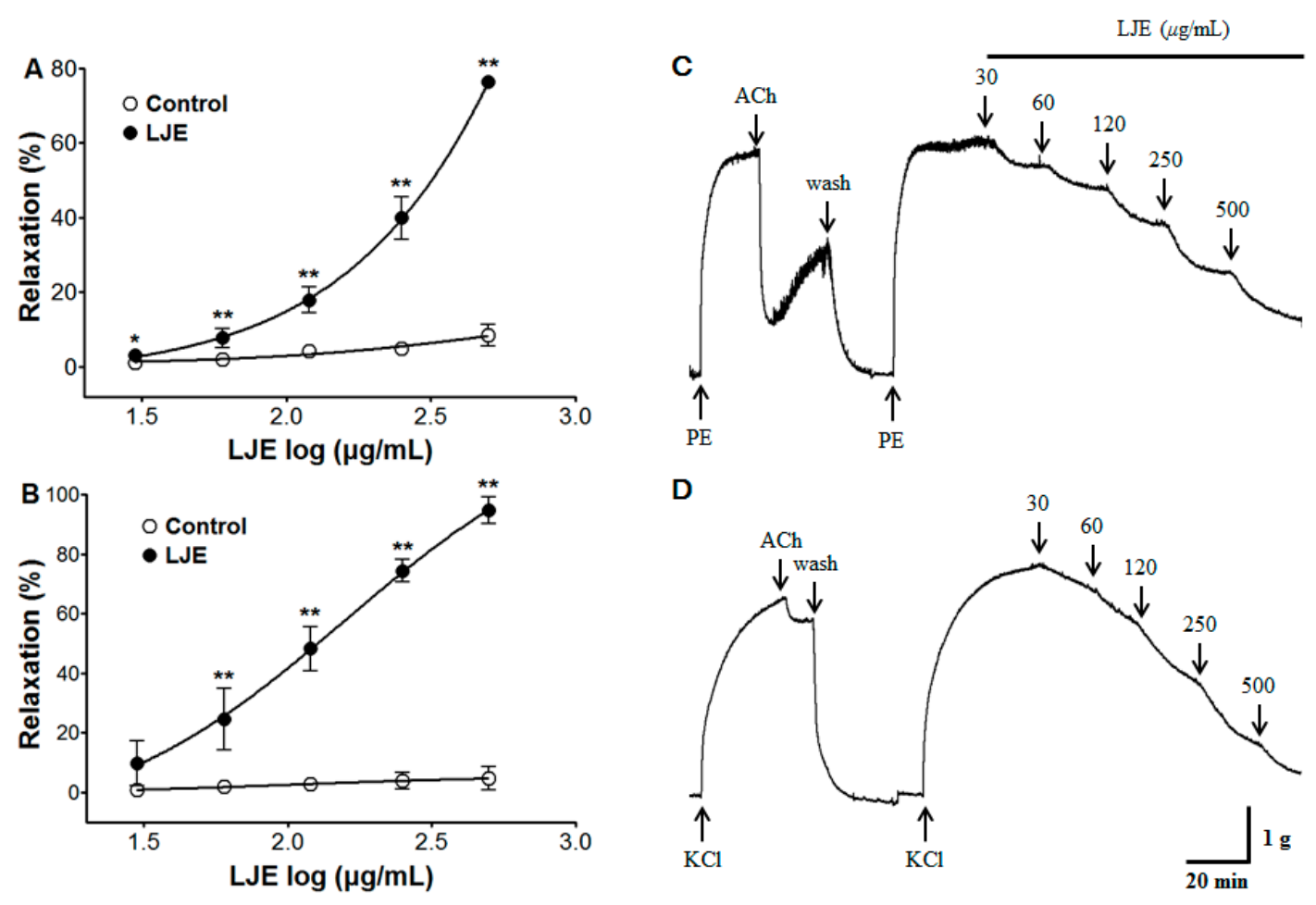

Figure 1. The concentration-dependent relaxation effect of LJE $(30-500 \mu \mathrm{g} / \mathrm{mL})$ in the presence or absence (control) of LJE on PE $(1 \mu \mathrm{M})(\mathbf{A})$ or $\mathrm{KCl}(60 \mathrm{mM})(\mathbf{B})$ pre-contracted aortic rings. The traces of LJE induced-relaxant in endothelium-intact aortic rings pre-contraction by $\mathrm{PE}(\mathbf{C})$ or $\mathrm{KCl}(\mathbf{D})$. Values are expressed as mean $\pm \operatorname{SEM}(n=4-7)$. $* p<0.05, * * p<0.01$ vs. control.

Few studies have evaluated the vasorelaxant effect of the genus Ligusticum (Umbelliferae). The ethanol extract of $L$. wallichii showed a maximal relaxant effect at $3000 \mu \mathrm{g} / \mathrm{mL}$ in isolated rat aorta [17]. The maximal relaxant effect of $L$. sinensis and $L$. jeholense essential oil was $76.7 \%-99.6 \%$ and $80.8 \%$ at $990 \mu \mathrm{g} / \mathrm{mL}$, respectively [16]. In the study, the maximal relaxant effect of LJE was observed at $500 \mu \mathrm{g} / \mathrm{mL}$. Considering the increasing interest in traditional medicines, L. jeholense may represent a potential candidate for the treatment of hypertension at lower doses than other genus Ligusticum therapeutics. 


\subsection{Effect of LJE on Endothelium-Intact or Endothelium-Denuded Aortic Rings Pre-Contracted with} $\mathrm{PE}$ or $\mathrm{KCl}$

We investigated the concentration-dependent vasorelaxant effect of LJE $(30-500 \mu \mathrm{g} / \mathrm{mL})$ on endothelium-intact and endothelium-denuded aortic rings pre-contracted with $\mathrm{PE}(1 \mu \mathrm{M})$ or $\mathrm{KCl}(60 \mathrm{mM})$ in standard $\mathrm{KH}$ buffer. The vasorelaxant effect of LJE was calculated as a percentage of the relaxation in response to $\mathrm{PE}$ and $\mathrm{KCl}$ on the aortic rings.

LJE caused concentration-dependent relaxation in both endothelium-intact and endothelium-denuded aortic rings pre-contracted by $\mathrm{PE}$ or $\mathrm{KCl}$ treatment. The maximal relaxant effect on PE-induced contraction was $76.4 \pm 1.2 \%$ and $75.2 \pm 2.0 \%$ for endothelium-intact and endothelium-denuded aortic rings, respectively (Figure 2). And for $\mathrm{KCl}$-induced contraction, the maximal relaxant effect was $94.6 \pm 4.5 \%$ and $96.3 \pm 3.6 \%$ for endothelium-intact and endothelium-denuded aortic rings, respectively (Figure 2).

The endothelial cell plays a major role in the vascular system, as it secretes regulated mediators or alters surface protein expressions that are vital for human homeostasis [18]. LJE caused concentration-dependent relaxation regardless of endothelial function. These results suggested that the vasorelaxant effect of LJE was endothelium independent.
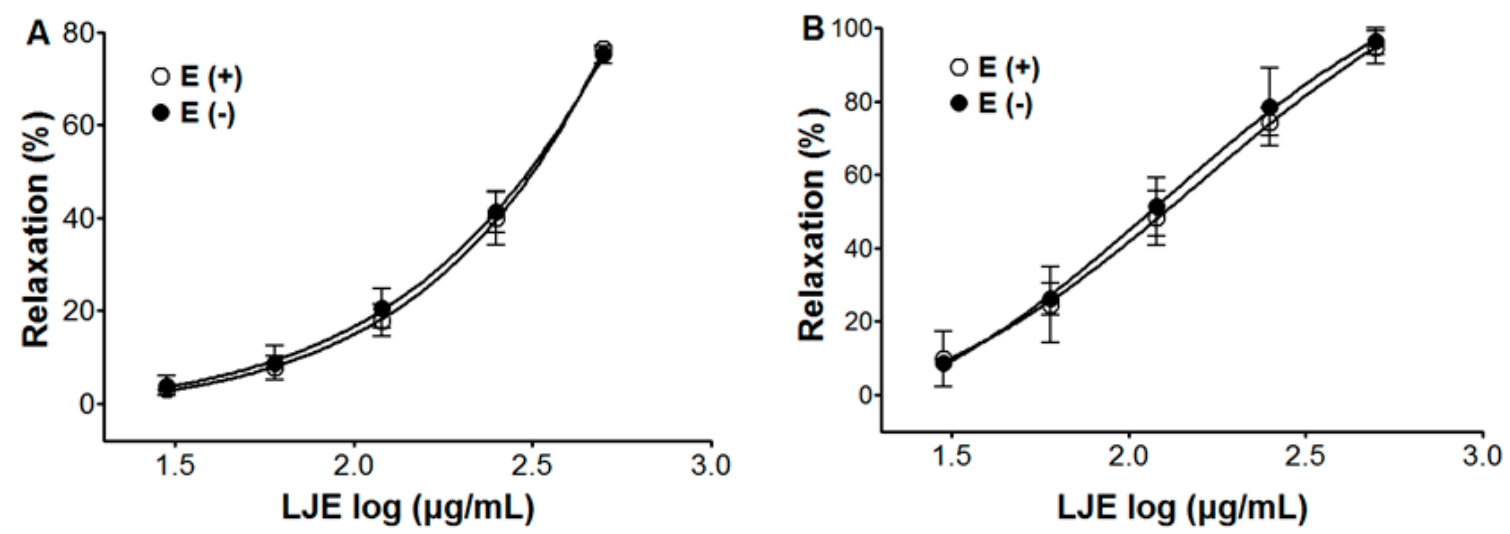

Figure 2. Concentration-dependent relaxant effect of LJE on PE $(1 \mu \mathrm{M})(\mathbf{A})$ or $\mathrm{KCl}(60 \mathrm{mM})$; (B) pre-contracted aortic rings with $[(\mathrm{E}+)]$ or without $\left[\left(\mathrm{E}^{-}\right)\right]$endothelium. Values are expressed as mean $\pm \operatorname{SEM}(n=5-7)$.

\subsection{Effect of LJE on Endothelium-Intact Aortic Rings Pre-Incubated with l-N Nitro Arginine Methyl} Ester (L-NAME), 1-H-[1,2,4]-Oxadiazolo-[4,3- $\alpha]$-quinoxalin-1-one (ODQ), or Methylene Blue (MB)

We investigated the vasorelaxant effect of LJE $(30-500 \mu \mathrm{g} / \mathrm{mL})$ on the nitric oxide (NO) synthesis pathway in endothelium-intact aortic rings that were pre-incubated with 1-NAME $(10 \mu \mathrm{M})$ for 20 min before PE $(1 \mu \mathrm{M})$ pre-contraction. We investigated the vasorelaxant effect of LJE on the NO-cyclic guanosine monophosphate (cGMP) pathway in endothelium-intact aortic rings that were pre-incubated with ODQ $(10 \mu \mathrm{M})$ or MB $(10 \mu \mathrm{M})$ for 20 min before PE pre-contraction. Compared to the control, the vasorelaxant effect of LJE was calculated as a percentage of the relaxation in response to L-NAME, ODQ, or MB pre-treatment on the aortic rings.

Incubation with L-NAME, ODQ, or MB did not affect LJE-induced relaxation of endothelium-intact aortic rings pre-contracted by PE treatment. In the presence and absence of L-NAME, the maximal 
relaxant effect was $76.4 \pm 1.2 \%$ and $76.0 \pm 2.7 \%$, respectively (Figure 3 ). The maximal relaxant effect in the presence and absence of ODQ was $72.0 \pm 5.0 \%$ and $76.4 \pm 1.2 \%$, respectively (Figure 3). In incubation with $\mathrm{MB}$, a maximal relaxant effect was of $73.1 \pm 3.9 \%$ compared to control $76.4 \pm 1.2 \%$ (Figure 3).

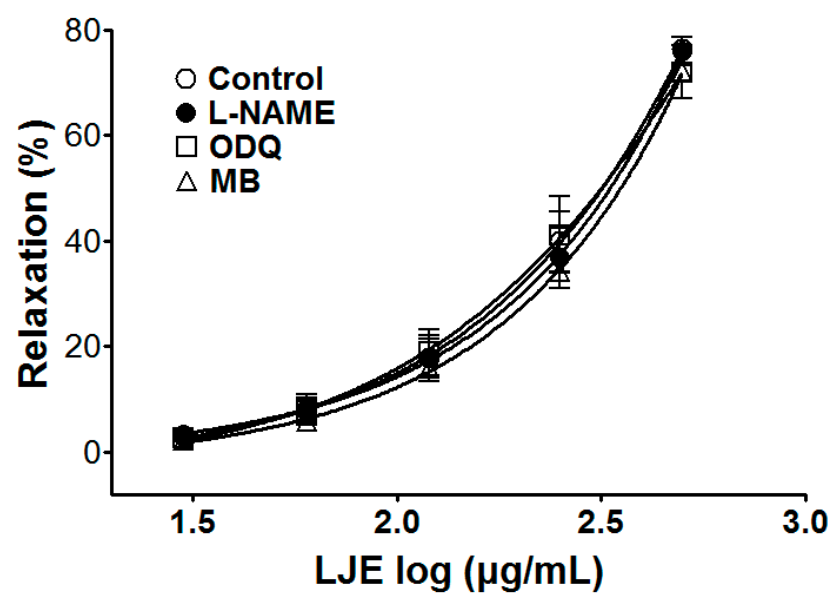

Figure 3. Concentration-dependent relaxant effect of LJE on PE $(1 \mu \mathrm{M})$-pre-contracted aortic rings in the presence or absence (control) of L-NAME $(10 \mu \mathrm{M})$, ODQ $(10 \mu \mathrm{M})$, or $\mathrm{MB}(10 \mu \mathrm{M})$. Values are expressed as mean $\pm \operatorname{SEM}(n=5-7)$.

Endothelium induces not only vasoconstriction via the generation of endothelin, prostanoids, and conversion of angiotensin I to angiotensin II at the endothelial surface, but also vasorelaxation via the secretion of NO, prostacyclin, and endothelium-derived hyperpolarizing factor (EDHF). In endothelial cells, NO is generated from L-arginine by endothelial NO synthase (eNOS) activation, which is stimulated by the calcium-calmodulin complex. In the vascular smooth cells, NO gas activates soluble guanylate cyclase (sGC), which increases cyclic GMP (cGMP) and leads to cGMP-mediated vasodilation [19-21].

To investigate endothelium-derived vasorelaxation, the various inhibitors of LJE-induced vasorelaxation were used. Pre-treatment with L-NAME (NOS inhibitor), ODQ, or MB (sGC inhibitor) did not affect the vasorelaxant effects of LJE. These results suggested that the vasorelaxant effect of LJE did not have a relationship with the direct NO pathway, NO-cGMP pathway.

\subsection{Effect of LJE on Endothelium-Intact Aortic Rings Pre-Incubated with Indomethacin}

We examined the vasorelaxant effect of LJE $(30-500 \mu \mathrm{g} / \mathrm{mL})$ on the prostacyclin pathway in endothelium-intact aortic rings, that were pre-incubated with indomethacin $(1 \mu \mathrm{M})$ for 20 min before PE $(1 \mu \mathrm{M})$ pre-contraction. Compared to the control, the vasorelaxant effect of LJE was calculated as a percentage of the relaxation in response to indomethacin pre-treatment on the aortic rings.

Incubation with indomethacin did not affect LJE-induced relaxation of endothelium-intact aortic rings pre-contracted by PE treatment. In the presence and absence of indomethacin, the maximal relaxant effect was $72.6 \pm 5.5 \%$ and $76.4 \pm 1.2 \%$, respectively (Figure 4 ).

Prostacyclin, which is produced by cyclooxygenase-1 from arachidonic acid, increases $3{ }^{\prime}-5^{\prime}$-cyclic adenosine monophosphate (cAMP), which leads to vascular smooth muscle relaxation as a result [22]. Pre-treatment with indomethacin (a non-selective cyclooxygenase inhibitor) did not affect the 
vasorelaxant effects of LJE. This result suggested that the vasorelaxant effect of LJE did not have a relationship with vascular prostacyclin pathway.

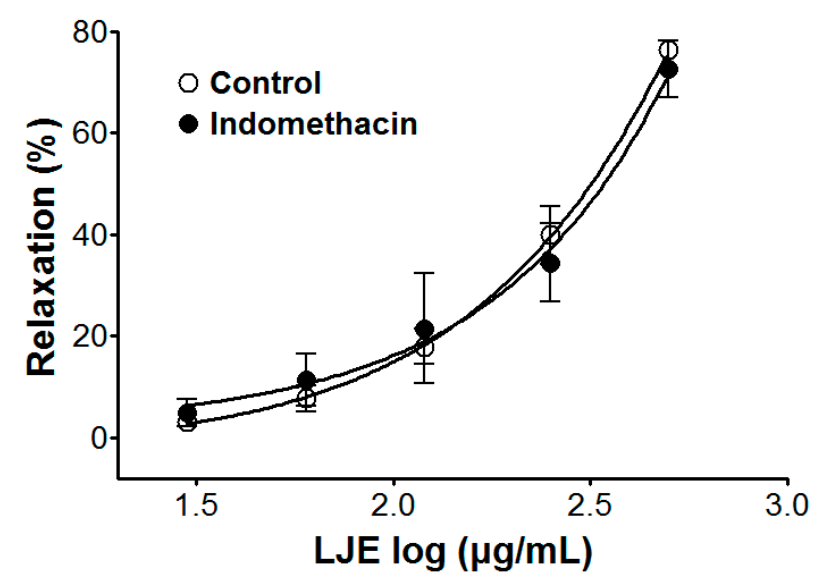

Figure 4. Cumulative concentration-response curves to LJE on PE $(1 \mu \mathrm{M})$-pre-contracted aortic rings in the presence or absence (control) of indomethacin $(1 \mu \mathrm{M})$. Values are expressed as mean $\pm \operatorname{SEM}(n=4-7)$.

\subsection{Effect of LJE on Endothelium-Intact Aortic Rings Pre-Incubated with Atropine}

We examined the vasorelaxant effect of LJE $(30-500 \mu \mathrm{g} / \mathrm{mL})$ from stimulation of muscarinic receptors in endothelium-intact aortic rings, that were pre-incubated with atropine $(1 \mu \mathrm{M})$ for $20 \mathrm{~min}$ before PE $(1 \mu \mathrm{M})$ pre-contraction. Compared to the control, the vasorelaxant effect of LJE was calculated as a percentage of the relaxation in response to atropine pre-treatment on the aortic rings.

Incubation with atropine did not affect LJE-induced relaxation of endothelium-intact aortic rings pre-contracted by $\mathrm{PE}$ treatment. In the presence and absence of atropine, the maximal relaxant effect was $70.9 \pm 3.8 \%$ and $76.4 \pm 1.2 \%$, respectively (Figure 5). Pre-treatment with atropine (a muscarinic receptor antagonist) did not affect the vasorelaxant effects of LJE. This result suggested that LJE was not associated with the muscarinic receptor.

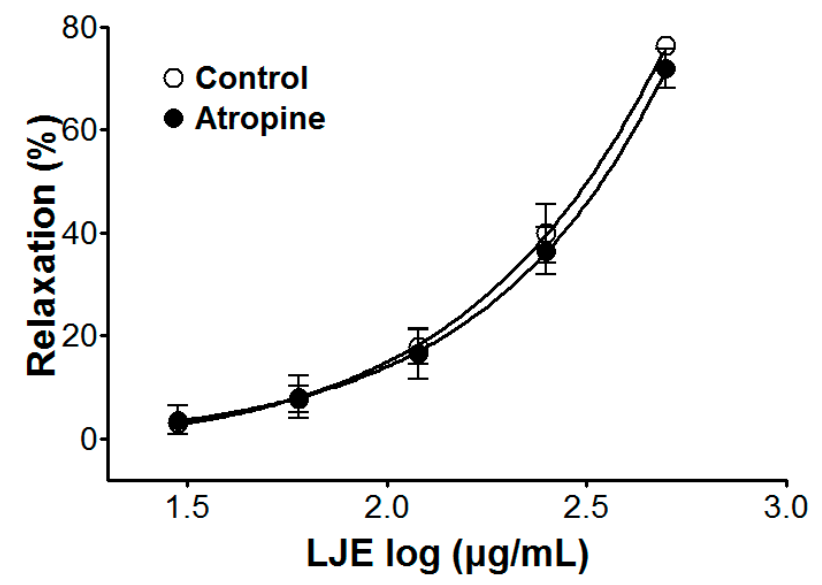

Figure 5. Cumulative concentration-response curves to LJE on PE $(1 \mu \mathrm{M})$-pre-contracted aortic rings in the presence or absence (control) of atropine $(1 \mu \mathrm{M})$. Values are expressed as mean $\pm \operatorname{SEM}(n=5-7)$. 


\subsection{Effect of LJE on Endothelium-Intact Aortic Rings Pre-Incubated with Various $K^{+}$Channel Blockers}

We examined the vasorelaxant effect of LJE $(30-500 \mu \mathrm{g} / \mathrm{mL})$ in endothelium-intact aortic rings, that were pre-incubated with a $\mathrm{K}^{+}$channel blocker such as tetraethylammonium (TEA, $5 \mathrm{mM}$ ), glibenclamide $(10 \mu \mathrm{M})$, or 4-aminopyridine (4-AP, $1 \mathrm{mM})$ for $20 \mathrm{~min}$ before PE $(1 \mu \mathrm{M})$ pre-contraction. Compared to the control, the vasorelaxant effect of LJE was calculated as a percentage of the relaxation in response to $\mathrm{K}^{+}$channel blockers pre-treatment on the aortic rings.

The vasorelaxant effects of LJE on PE pre-contracted endothelium-intact aortic rings were altered by incubation with $\mathrm{K}^{+}$channel blockers including glibenclamide or 4-AP. In the presence of glibenclamide or 4-AP, the LJE-induced maximal relaxant effect was of $68.1 \pm 4.7 \%$ and $60.0 \pm 3.4 \%$, respectively (Figure 6). Incubation with TEA did not affect LJE-induced relaxation of endothelium-intact aortic rings pre-contracted by PE treatment (Figure 6).

In this study, removal of endothelial function or pre-treatment with L-NAME did not inhibit the relaxant effects of LJE. Thus, we concluded that the vasorelaxant effects of LJE might be due to the vascular smooth muscle.

The contraction and relaxation of vascular smooth muscle is regulated by the membrane potential through changes in $\mathrm{K}^{+}$channel activity. The $\mathrm{K}^{+}$channel activity is a main mechanism of vasoconstriction and vasodilation. In vascular smooth cells, the vascular activity is regulated by the membrane potential through changes in $\mathrm{K}^{+}$channel activity, which changes activity of voltage-dependent $\mathrm{Ca}^{2+}$ channels [23]. To investigate the possibility that the vasorelaxant effects of $\mathrm{LJE}$ are mediated via $\mathrm{K}^{+}$channels, various $\mathrm{K}^{+}$channel blockers such as glibenclamide (KATP channels blocker, a highly selective blocker of ATP-sensitive $\mathrm{K}^{+}$channels), TEA ( $\mathrm{K}_{\mathrm{Ca}}$ channels blocker, a blocker of big $\mathrm{Ca}^{2+}$-activated $\mathrm{K}^{+}$channels), and 4-AP ( $\mathrm{K}_{\mathrm{V}}$ channels blocker, a predominant blocker of voltage-gated $\mathrm{K}^{+}$channels) were used [24]. The vasorelaxant effect of LJE was partly attenuated by pre-treatment with glibenclamide or 4-AP as $\mathrm{K}^{+}$channel blocker. We found that the vasorelaxant effects of LJE on the rat aortic rings are related to $\mathrm{K}^{+}$channels such as $\mathrm{K}_{\mathrm{ATP}}$ channels and $\mathrm{K}_{\mathrm{V}}$ channels.

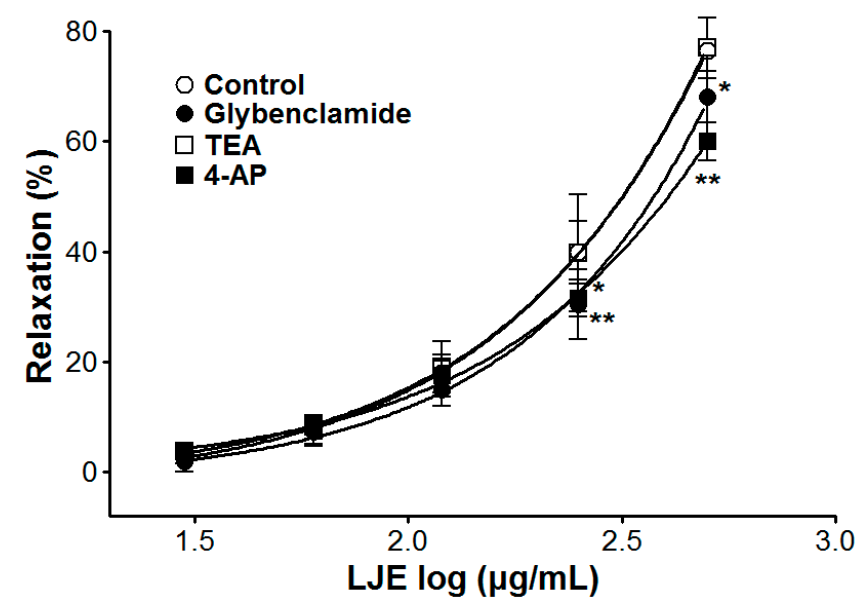

Figure 6. Cumulative concentration-response curves to LJE on endothelium-intact aortic rings pre-contracted with $\mathrm{PE}(1 \mu \mathrm{M})$ in the presence or absence (control) of glibenclamide $(10 \mu \mathrm{M})$, TEA $(5 \mathrm{mM})$, or $4-\mathrm{AP}(1 \mathrm{mM})$. Values are expressed as mean $\pm \mathrm{SEM}(n=5-7)$. $* p<0.05, * * p<0.01$ vs. control. 
2.7. Effect of LJE on Extracellular $\mathrm{Ca}^{2+}$-Induced Contraction (via Receptor-Operative Ca ${ }^{2+}$ Channels or Voltage-Dependent $\mathrm{Ca}^{2+}$ Channels)

We examined the vasorelaxant effect of LJE $(120-500 \mu \mathrm{g} / \mathrm{mL})$ on extracellular $\mathrm{Ca}^{2+}$-induced contractions via receptor-operative $\mathrm{Ca}^{2+}$ channels (ROCCs) and voltage-dependent $\mathrm{Ca}^{2+}$ channels (VDCCs), by $\mathrm{PE}$ or $\mathrm{KCl}$ pre-treatment, respectively. We tested the contraction response induced by calcium chloride $\left(\mathrm{CaCl}_{2}, 0.3-10 \mathrm{mM}\right)$ in the endothelium-denuded aortic rings by PE $(1 \mu \mathrm{M})$ or $\mathrm{KCl}$ $(60 \mathrm{mM})$ pre-contraction in $\mathrm{Ca}^{2+}$-free $\mathrm{KH}$ buffer with and without (control) LJE preincubation for $10 \mathrm{~min}$. Compared to the control, the contraction responses induced by $\mathrm{CaCl}_{2}$ were calculated as a percentage in the presence and absence (control) of LJE pre-treatment.

In $\mathrm{Ca}^{2+}$-free $\mathrm{KH}$ buffer, the cumulative addition of $\mathrm{CaCl}_{2}(0.3-10 \mathrm{mM})$ induced progressively increased tension in the rat thoracic aorta rings. As shown in Figure 7, LJE $(120-500 \mu \mathrm{g} / \mathrm{mL})$ pre-incubation significantly inhibited the contractions induced by extracellular $\mathrm{CaCl}_{2}(10 \mathrm{mM})$ and the contraction at LJE $(500 \mu \mathrm{g} / \mathrm{mL})$ concentration was decreased to $0.00 \pm 0.10 \mathrm{~g}$ and $-0.18 \pm 0.16 \mathrm{~g} v \mathrm{~s}$. the control group $1.47 \pm 0.21 \mathrm{~g}$ and $1.30 \pm 0.13 \mathrm{~g}$, in cells pre-contracted by $\mathrm{PE}$ and $\mathrm{KCl}$, respectively (Figure 7).

The contraction and relaxation of vascular smooth muscle is regulated by $\mathrm{Ca}^{2+}$ entry from the extracellular space through ROCCs or VDCCs in the plasma membrane, through $\mathrm{Ca}^{2+}$ release from intracellular $\mathrm{Ca}^{2+}$ stores (sarcoplasmic reticulum), protein kinase $\mathrm{C}$ (PKC) activation, and a $\mathrm{Ca}^{2+}$ sensitization mechanism [25]. PE induced the influx of extracellular $\mathrm{Ca}^{2+}$ by activating ROCCs and $\mathrm{KCl}$ induced $\mathrm{Ca}^{2+}$ influx through VDCCs [26]. LJE inhibited vasoconstriction induced by $\mathrm{Ca}^{2+}$ supplementation in the aortic rings that were pre-contracted with $\mathrm{PE}$ or $\mathrm{KCl}$ in $\mathrm{Ca}^{2+}$-free $\mathrm{KH}$ buffer. These results suggested that LJE significantly inhibited the entry of extracellular $\mathrm{Ca}^{2+}$ via ROCCs or VDCCs activated by PE or KCl.
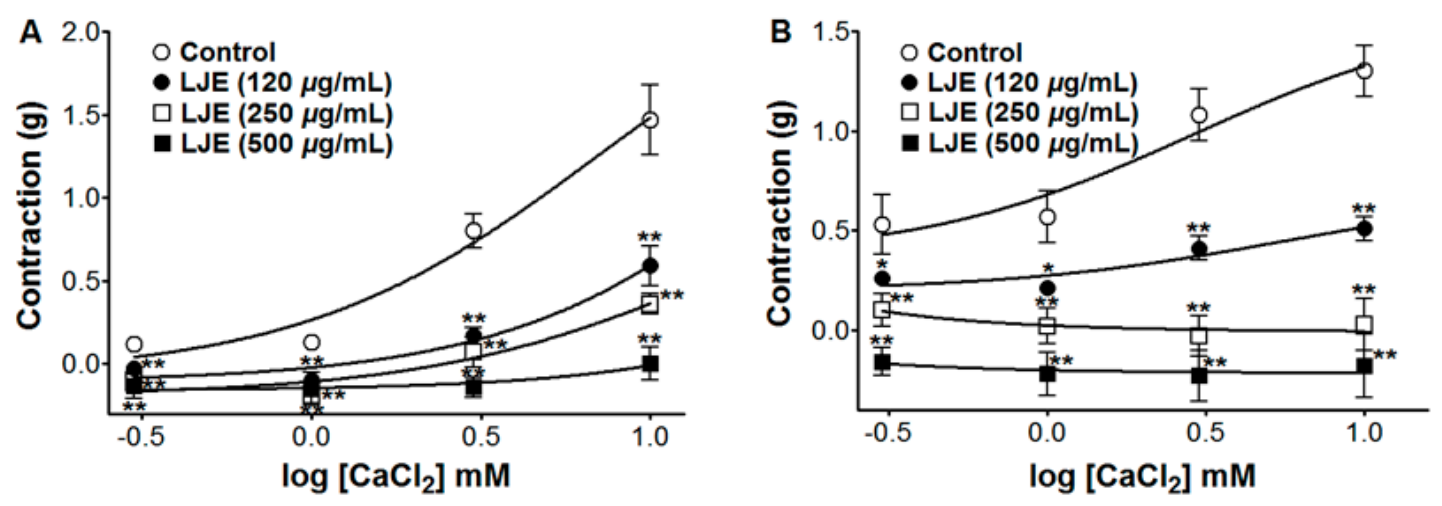

Figure 7. Inhibitory effect of LJE $(120-500 \mu \mathrm{g} / \mathrm{mL})$ on the contraction induced by extracellular $\mathrm{Ca}^{2+}$ in endothelium-denuded rat thoracic aorta rings that were pre-contracted with PE $(1 \mu \mathrm{M})(\mathbf{A})$ or $\mathrm{KCl}(60 \mathrm{mM})(\mathbf{B})$ in the presence or absence (control) of LJE. Values are expressed as mean $\pm \operatorname{SEM}(n=4)$. ${ }^{*} p<0.05, * * p<0.01 v s$. control.

\subsection{Effect of LJE and SK \& F96365 on PE-Induced Contraction in the Presence of Nifedipine}

The effect of LJE on $\mathrm{Ca}^{2+}$ influx through ROCCs was investigated by determining the effect of LJE $(500 \mu \mathrm{g} / \mathrm{mL})$ and the ROCCs blocker SK \& F $96365(50 \mu \mathrm{M})$ on PE $(1 \mu \mathrm{M})$-induced contraction in the 
presence of the VDCCs blocker nifedipine $(10 \mu \mathrm{M})$. PE was applied twice in the presence of nifedipine; the aortic rings were treated with LJE or SK \& F 96365 before the second application of PE.

Nifedipine inhibited the contraction induced by PE. Further inhibition was observed after the application of LJE $(500 \mu \mathrm{g} / \mathrm{mL})$ or SK \& F 96365 (Figure 8). Nifedipine is an inhibitor of voltage-gated $\mathrm{Ca}^{2+}$ entry and SK \& F 96365 is a selective inhibitor of receptor-mediated $\mathrm{Ca}^{2+}$ entry [27]. A combination of SK \& F 96365 and nifedipine decreased further PE-induced contractions. As a result, nifedipine blocked VDCCs at first and SK \& F 96365 blocked ROCCs in sequence. Likewise, LJE $(500 \mu \mathrm{g} / \mathrm{mL})$ decreased PE-induced contractions in the presence of nifedipine, suggesting that LJE inhibits the entry of extracellular $\mathrm{Ca}^{2+}$ via ROCCs activated by PE.

Many compounds, including linoleic acid, ferulic acid, and beta-sitosterol, were isolated from L. jeholense root and rhizoma [15]. The main components of essential oil from this plant were reported to be $m$-diaminobenzene $(68.2 \%)$, ligustilide $(10.1 \%), p$-vinylguaiacol $(3.5 \%)$, apiol $(2.0 \%)$ [7], or $\beta$-phellandrene (33.3\%) [5]. The vasorelaxant effects of these few compounds have been reported previously. Ferulic acid inhibits angiotensin II-induced constriction of the vascular smooth muscle cells by regulating cell cycle progression [28]. Linoleic acid brings about vasorelaxation and hyperpolarization by activating $\mathrm{Na}^{+} / \mathrm{K}^{+}$-ATPase pumps [29]. Our results also showed that LJE-induced vasorelaxation was related to ATP-sensitive and voltage-sensitive $\mathrm{K}^{+}$channels ( $\mathrm{K}_{\mathrm{ATP}}$ and $\mathrm{K}_{\mathrm{V}}$ channels). It was found in previous studies that the vasorelaxant effects of ligustilide were not altered by endothelium removal or inhibition of adenylate cyclase, sGC [8]. Likewise, in our study, it was found that the vasorelaxant effects of LJE were endothelium-independent and not related to the NO-cGMP pathway. Hence, it is likely that linoleic acid, ferulic acid, or ligustilide could be responsible for the vasorelaxant effects of this plant. L. jeholense consists of various compounds, and further investigation is required to examine the mechanism of active compounds of this plant in rat aortic rings.
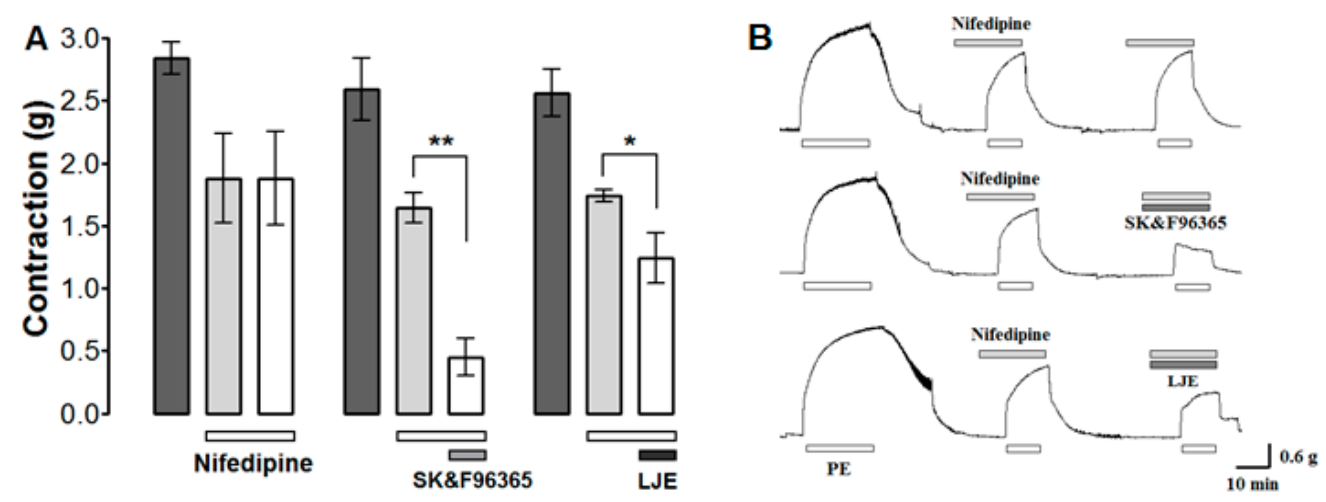

Figure 8. The effects (A) and traces (B) of LJE $(500 \mu \mathrm{g} / \mathrm{mL})$ and SK \& F96365 $(50 \mu \mathrm{M})$ in the presence of nifedipine $(10 \mu \mathrm{M})$ on PE-induced contraction. Values are expressed as mean $\pm \operatorname{SEM}(n=4) . * p<0.05, * * p<0.01$.

\section{Experimental Section}

\subsection{Chemicals and Drugs}

Modified $\mathrm{KH}$ buffer powder, $\mathrm{PE}, \mathrm{KCl}, \mathrm{L}-\mathrm{NAME}, \mathrm{ODQ}, \mathrm{MB}$, indomethacin, atropine, TEA, glibenclamide, 4-AP, $\mathrm{CaCl}_{2}$, ethylene glycol bis (2-aminoethylether)- $N, N, N^{\prime}, N^{\prime}$-tetraacetic acid (EGTA), 
SK \& F96365, nifedipine, and dimethyl sulfoxide (DMSO) were purchased from Sigma Aldrich (St. Louis, MO, USA). All other reagents were of analytical purity.

\subsection{Plant Material and Extraction}

L. jeholense root and rhizoma, were collected between $37^{\circ} 13^{\prime} \mathrm{N}$ to $37^{\circ} 21^{\prime} \mathrm{N}$ latitude and $128^{\circ} 43^{\prime} \mathrm{E}$ to $128^{\circ} 55^{\prime} \mathrm{E}$ longitude in Jeongseon, Gangwon Province, Republic of Korea, in August 2014. Professor Hocheol Kim of Kyung Hee University identified the plant. A voucher specimen of the plant (VS14080201) was deposited in the herbarium of the College of Korean Medicine, Kyung Hee University, Seoul, Republic of Korea. Dried L. jeholense root and rhizoma (100.0 g) was extracted three times with $1 \mathrm{~L} 100 \% \mathrm{MeOH}$ for $3 \mathrm{~h}$ in a reflux apparatus at $70 \pm 5{ }^{\circ} \mathrm{C}$. After filtration, the extract was evaporated in a rotary vacuum evaporator (N-N series, EYELA, Tokyo, Japan) at $60{ }^{\circ} \mathrm{C}$ and lyophilized in a freeze-dryer (Operon ${ }^{\mathrm{TM}}$, Seoul, Korea) to obtain a dark brown powder $(20.9 \mathrm{~g})$ of crude extract. LJE powder was accurately weighed $(0.1 \mathrm{~g})$, suspended in $1 \mathrm{~mL} \mathrm{KH}$ buffer, and placed into an ultrasonic device for $1 \mathrm{~min}$ for solubilization. The powder was completely dissolved, and the color was light brown.

\subsection{Animals}

We used male Sprague-Dawley rats (weight, 240-260 g; Raonbio, Yongin, Gyeonggi Province, Korea). All animal procedures were conducted according to the animal welfare guidelines and were approved [KHUASP(SE)-15-013] by the Kyung Hee University Institutional Animal Care and Use Committee. All rats were acclimated under standard laboratory conditions $\left(22 \pm 2{ }^{\circ} \mathrm{C}\right.$; lighting, 07:00-19:00) and food and water were given ad libitum.

\subsection{Preparation of Rat Aortic Rings}

Rats were anesthetized by exposure to ether, the thoracic aorta was removed and immersed in $\mathrm{KH}$ buffer [composition (mM): $\mathrm{NaCl}, 118.0 ; \mathrm{KCl}, 4.7 ; \mathrm{MgSO}_{4}, 1.2 ; \mathrm{KH}_{2} \mathrm{PO}_{4}, 1.2 ; \mathrm{CaCl}_{2}, 2.5 ; \mathrm{NaHCO}_{3}$, 25.0; and glucose, 11.1; $\mathrm{pH} 7.4]$, and then aerated with a gas mixture of $95 \% \mathrm{O}_{2}-5 \% \mathrm{CO}_{2}$ at $37{ }^{\circ} \mathrm{C}$. After carefully removing the connective tissue and fat surrounding the aorta, the aorta was cut into 2-mm-long rings and suspended in organ chambers containing $10 \mathrm{~mL} \mathrm{KH}$ buffer at $37^{\circ} \mathrm{C}$. The rings were suspended between two tungsten stirrups and one stirrup was connected to an isometric force transducer (Grass Instrument Co., West Warwick, RI, USA). The aortic ring segments were incubated under no tension for $30 \mathrm{~min}$ and left to equilibrate for $1 \mathrm{~h}$ at an optimal resting tension of $1.2 \mathrm{~g}$. The $\mathrm{KH}$ buffer was refreshed every 15-20 min during the equilibration period for $90 \mathrm{~min}$. The changes in tension of the aortic rings were recorded using isometric transducers connected to a data acquisition system (PowerLab, ADI instrument Co., Bella Vista, New South Wales, Australia). When necessary, the endothelium layer was removed by gently rubbing inside the lumen using a thin polyethylene stick. The presence of functional endothelium was checked by the ability of ACh $(10 \mu \mathrm{M})$ to induce more than $80 \%$ relaxation in PE $(1 \mu \mathrm{M})$-contracted aorta rings. Endothelium-denudation was considered effectively removed when $\mathrm{ACh}$ caused less than $10 \%$ relaxation. $\mathrm{Ca}^{2+}$-free $\mathrm{KH}$ buffer was prepared by 
removing $\mathrm{CaCl}_{2}$ and adding EGTA $(1 \mathrm{mM})$. The antagonist and inhibitor concentrations were selected in the same manner as described previously [30,31].

\subsection{Data Analysis}

Results are expressed as mean \pm standard error of mean (SEM). Statistical comparisons were made using Student's $t$-test. All statistical analyses were performed by using SPSS (version 21.0) statistical analysis software (SPSS Inc., Chicago, IL, USA). $P$ values less than 0.05 were considered statistically significant.

\section{Conclusions}

In conclusion, (1) the vasorelaxant effects of LJE were not related to the direct NO pathway, NO-cGMP pathway, vascular prostacyclin ( $\mathrm{PGI}_{2}$ ) pathway, or muscarinic receptors transduction pathway; (2) $\mathrm{K}^{+}$ channels were partly related to LJE-induced vasorelaxation; and (3) LJE relaxed the aortic rings by blocking the entry of extracellular $\mathrm{Ca}^{2+}$ via ROCCs and VDCCs.

\section{Acknowledgments}

This study was supported by a grant from the High Value-added Food Technology Development Program, Korea Institute of Planning \& Evolution for Technology in Food, Agriculture, Forestry \& Fisheries (314071-03-1-HD020).

\section{Author Contributions}

B.K. and K.S.C. performed the extraction and experiments. K.L., I.H., Y.B. and H.K. analyzed the data. H.-Y.C. has corrected the final version of the manuscript.

\section{Conflicts of Interest}

The authors declare no conflict of interest.

\section{References}

1. Handler, J. Quality of Life and Antihypertensive Drug Therapy. J. Clin. Hypertens. 2005, 7, 274-285.

2. Alaerts, G.; Merino-Arevalo, M.; Dumarey, M.; Dejaegher, B.; Noppe, N.; Matthijs, N.; Smeyers-Verbeke, J.; Vander Heyden, Y. Exploratory Analysis of Chromatographic Fingerprints to Distinguish Rhizoma Chuanxiong and Rhizoma Ligustici. J. Chromatogr. A 2010, 1217, 7706-7716.

3. Xia, M.; Qian, L.; Zhou, X.; Gao, Q.; Bruce, I.C.; Xia, Q. Endothelium-Independent Relaxation and Contraction of Rat Aorta Induced by Ethyl Acetate Extract from Leaves of Morus alba (L.). J. Ethnopharmacol. 2008, 120, 442-446.

4. Ibarra-Alvarado, C.; Rojas, A.; Mendoza, S.; Bah, M.; Gutierrez, D.M.; Hernandez-Sandoval, L.; Martinez, M. Vasoactive and Antioxidant Activities of Plants used in Mexican Traditional Medicine for the Treatment of Cardiovascular Diseases. Pharm. Biol. 2010, 48, 732-739. 
5. Sieniawska, E.; Baj, T.; Ulewicz-Magulska, B.; Wesolowski, M.; Glowniak, K. The Essential Oils from Ligusticum mutellina of Polish Origin and the Chemical Relationship of its Root Essential Oil with Other Ligusticum Species. Biochem. Syst. Ecol. 2013, 49, 125-130.

6. Yook, C.; Kang, C.; Inn, M.; Kim, K.; Kim, C. The Essential Oils of Ligusticum tenuissimum roots. Yakhak Hoeji 1997, 41, 273-276.

7. Wang, J.; Xu, L.; Yang, L.; Liu, Z.; Zhou, L. Composition, Antibacterial and Antioxidant Activities of Essential Oils from Ligusticum sinense and L. jeholense (Umbelliferae) from China. Rec. Nat. Prod. 2011, 5, 314-318.

8. Chan, S.S.; Cheng, T.Y.; Lin, G. Relaxation Effects of Ligustilide and Senkyunolide A, Two Main Constituents of Ligusticum chuanxiong, in Rat Isolated Aorta. J. Ethnopharmacol. 2007, $111,677-680$.

9. Zang, J.L.; Zhou, Z.H.; Chen, R.Y.; Xie, F.Z.; Cheng, G.F.; Yu, D.Q.; Zhou, T.H. Study on Chemistry and Pharmacology of Genus Ligusticum. Chin. Pharm. J. 2002, 37, 654-657.

10. Xie, X.; Tian, Y.; Yin, S.; Lin, Y.; Tan, G. Anticancer Effects of Ligusticum chuanxiong Hort Alcohol Extracts on HS766T Cell. Afr. J. Tradit. Complement. Altern. Med. 2013, 10, 542-546.

11. Huang, J.; Lu, X.Q.; Zhang, C.; Lu, J.; Li, G.Y.; Lin, R.C.; Wang, J.H. Anti-Inflammatory Ligustilides from Ligusticum chuanxiong Hort. Fitoterapia 2013, 91, 21-27.

12. Guzman, J.D.; Evangelopoulos, D.; Gupta, A.; Prieto, J.M.; Gibbons, S.; Bhakta, S. Antimycobacterials from Lovage Root (Ligusticum officinale Koch). Phytother. Res. 2013, 27, 993-998.

13. Juarez-Reyes, K.; Angeles-Lopez, G.E.; Rivero-Cruz, I.; Bye, R.; Mata, R. Antinociceptive Activity of Ligusticum porteri Preparations and Compounds. Pharm. Biol. 2014, 52, 14-20.

14. Kim, B.W.; Koppula, S.; Park, S.Y.; Kim, Y.S.; Park, P.J.; Lim, J.H.; Kim, I.S.; Choi, D.K. Attenuation of Neuroinflammatory Responses and Behavioral Deficits by Ligusticum officinale (Makino) Kitag in Stimulated Microglia and MPTP-Induced Mouse Model of Parkinson's Disease. J. Ethnopharmacol. 2015, 164, 388-397.

15. Zhang, B.; Sun, J.M.; Chang, R.L.; Zhang, H. Studies on the Chemical Constituents of the Root and Rhizoma of Ligusticum jeholense. Zhong Yao Cai 2009, 32, 710-712.

16. Zhang, Y.; Chen, C.; Li, S.; Xu, H.; Li, D.; Wu, H.; Yang, H. Chemical Analysis and Observation on Vascular Activity of Essential Oil from Ligusticum sinensis, Conioselinum tataricum and Ligusticum jeholense. Chin. J. Exp. Tradit. Med. Form. 2011, 17, 159-164.

17. Kim, E.Y.; Kim, J.H.; Rhyu, M.R. Endothelium-Independent Vasorelaxation by Ligusticum wallichii in Isolated Rat Aorta: Comparison of a Butanolic Fraction and Tetramethylpyrazine, the Main Active Component of Ligusticum wallichii. Biol. Pharm. Bull. 2010, 33, 1360-1363.

18. Pearson, J.D. Normal Endothelial Cell Function. Lupus 2000, 9, 183-188.

19. Forstermann, U; Munzel, T. Endothelial Nitric Oxide Synthase in Vascular Disease: From Marvel to Menace. Circulation 2006, 113, 1708-1714.

20. Deanfield, J.E.; Halcox, J.P.; Rabelink, T.J. Endothelial Function and Dysfunction: Testing and Clinical Relevance. Circulation 2007, 115, 1285-1295.

21. Stankevicius, E.; Kevelaitis, E.; Vainorius, E.; Simonsen, U. Role of Nitric Oxide and Other Endothelium-Derived Factors. Medicina (Kaunas) 2003, 39, 333-341. 
22. Faraci, F.M.; Heistad, D.D. Regulation of the Cerebral Circulation: Role of Endothelium and Potassium Channels. Physiol. Rev. 1998, 78, 53-97.

23. Brayden, J.E. Potassium Channels in Vascular Smooth Muscle. Clin. Exp. Pharmacol. Physiol. 1996, 23, 1069-1076.

24. Novakovic, A.; Bukarica, L.G.; Kanjuh, V.; Heinle, H. Potassium Channels-Mediated Vasorelaxation of Rat Aorta Induced by Resveratrol. Basic Clin. Pharmacol. Toxicol. 2006, 99 , 360-364.

25. Webb, R.C. Smooth Muscle Contraction and Relaxation. Adv. Physiol. Educ. 2003, 27, 201-206.

26. Zhang, N.; Zou, H.; Jin, L.; Wang, J.; Zhong, M.F.; Huang, P.; Gu, B.Q.; Mao, S.L.; Zhang, C.; Chen, H. Biphasic Effects of Sodium Danshensu on Vessel Function in Isolated Rat Aorta. Acta Pharmacol. Sin. 2010, 31, 421-428.

27. Soergel, D.G.; Yasumoto, T.; Daly, J.W.; Gusovsky, F. Maitotoxin Effects are Blocked by SK \& F 96365, an Inhibitor of Receptor-Mediated Calcium Entry. Mol. Pharmacol. 1992, 41, 487-493.

28. Hou, Y.Z.; Yang, J.; Zhao, G.R.; Yuan, Y.J. Ferulic Acid Inhibits Vascular Smooth Muscle Cell Proliferation Induced by Angiotensin II. Eur. J. Pharmacol. 2004, 499, 85-90.

29. Pomposiello, S.I.; Alva, M.; Wilde, D.W.; Carretero, O.A. Linoleic Acid Induces Relaxation and Hyperpolarization of the Pig Coronary Artery. Hypertension 1998, 31, 615-620.

30. Lee, K.; Ham, I.; Yang, G.; Lee, M.; Bu, Y.; Kim, H.; Choi, H.Y. Vasorelaxant Effect of Prunus yedoensis Bark. BMC Complement. Altern. Med. Rev. 2013, 13, doi:10.1186/1472-6882-13-31.

31. Jones, R.D.; Ruban, L.N.; Morton, I.E.; Roberts, S.A.; English, K.M.; Channer, K.S.; Jones, T.H. Testosterone Inhibits the Prostaglandin F2alpha-Mediated Increase in Intracellular Calcium in A7r5 Aortic Smooth Muscle Cells: Evidence of an Antagonistic Action upon Store-Operated Calcium Channels. J. Endocrinol. 2003, 178, 381-393.

Sample Availability: Samples of the this plant are available from the authors.

(C) 2015 by the authors; licensee MDPI, Basel, Switzerland. This article is an open access article distributed under the terms and conditions of the Creative Commons Attribution license (http://creativecommons.org/licenses/by/4.0/). 